\title{
Multiplicatively P-functions and some new inequalities
}

\author{
Huriye Kadakal
}

Ministry of Education, Bulancak Bahçelievler Anatolian High School Bulancak, Giresun, Turkey

Received: 17 October 2018, Accepted: 30 October 2018

Published online: 10 december 2018.

\begin{abstract}
In this study, we present a new definition of convexity. This definition is the class of multiplicatively P-functions. Some new Hermite-Hadamard type inequalities are derived for this class functions. After that some applications to special means of real numbers are given. Ideas of this paper may stimulate further research. We should especially mention that the definition of multiplicatively P-function is given for the first time in the literature by us.
\end{abstract}

Keywords: Convex function, multiplicatively P-function, Hölder integral inequality and power-mean integral inequality, HermiteHadamard type inequality.

\section{Preliminaries and fundamentals}

Definition 1. A function $f: I \subseteq \mathbb{R} \rightarrow \mathbb{R}$ is said to be convex if the inequality

$$
f(t x+(1-t) y) \leq t f(x)+(1-t) f(y)
$$

is valid for all $x, y \in I$ and $t \in[0,1]$. If this inequality reverses, then the function $f$ is said to be concave on interval $I \neq \emptyset$.

This definition is well known in the literature. Convexity theory has appeared as a powerful technique to study a wide class of unrelated problems in pure and applied sciences.

One of the most important integral inequalities for convex functions is the Hermite-Hadamard inequality. The classical Hermite-Hadamard inequality provides estimates of the mean value of a continuous convex function $f:[a, b] \rightarrow \mathbb{R}$. The following double inequality is well known as the Hadamard inequality in the literature.

Definition 2. Let $f:[a, b] \rightarrow \mathbb{R}$ be a convex function, then the inequality

$$
f\left(\frac{a+b}{2}\right) \leq \frac{1}{b-a} \int_{a}^{b} f(x) d x \leq \frac{f(a)+f(b)}{2}
$$

is known as the Hermite-Hadamard inequality.

Some refinements of the Hermite-Hadamard inequality on convex functions have been extensively investigated by a number of authors (e.g., $[3,4,6,7])$ and the Authors obtained a new refinement of the Hermite-Hadamard inequality for convex functions.

Definition 3. A nonnegative function $f: I \subseteq \mathbb{R} \rightarrow \mathbb{R}$ is said to be P-function if the inequality

$$
f(t x+(1-t) y) \leq f(x)+f(y)
$$


holds for all $x, y \in I$ and $t \in(0,1)$. We will denote by $P(I)$ the set of $P$-functions on the interval $I$. Note that $P(I)$ contain all nonnegative convex and quasi-convex functions.

In [1], Dragomir et al. proved the following inequality of Hadamard type for class of $P$-functions.

Theorem 1. Let $f \in P(I), a, b \in I$ with $a<b$ and $f \in L[a, b]$. Then

$$
f\left(\frac{a+b}{2}\right) \leq \frac{2}{b-a} \int_{a}^{b} f(x) d x \leq 2[f(a)+f(b)] .
$$

Dragomir and Agarwal in [2] used the following lemma to prove Theorems.

Lemma 1. The following equation holds true:

$$
\frac{f(a)+f(b)}{2}-\frac{1}{b-a} \int_{a}^{b} f(x) d x=\frac{b-a}{2} \int_{0}^{1}(1-2 t) f^{\prime}(t a+(1-t) b) d t .
$$

In [5], U. S. Kirmaci used the following lemma to prove Theorems.

Lemma 2. Let $f: I^{*} \subset \mathbb{R} \rightarrow \mathbb{R}$ be a differentiable mapping on $I^{*}, a, b \in I^{*}$ (I* is the interior of $I$ ) with $a<b$. If $f^{\prime} \in L[a, b]$, then we haveThe following equation holds true:

$$
\frac{1}{b-a} \int_{a}^{b} f(x) d x-f\left(\frac{a+b}{2}\right)=(b-a)\left[\int_{0}^{\frac{1}{2}} t f^{\prime}(t a+(1-t) b) d t+\int_{\frac{1}{2}}^{1}(t-1) f^{\prime}(t a+(1-t) b) d t\right] .
$$

The main purpose of this paper is to establish new estimations and refinements of the Hermite-Hadamard inequality for functions whose derivatives in absolute value are multiplicatively $P$-function.

\section{Definition of multiplicatively $P$-functions and their some properties}

In this section, we begin by setting some algebraic properties for multiplicatively $P$-functions.

Definition 4. Let $I \neq \emptyset$ be an interval in $\mathbb{R}$. The function $f: I \rightarrow[0, \infty)$ is said to be multiplicatively P-function (or log-Pfunction), if the inequality

$$
f(t x+(1-t) y) \leq f(x) f(y)
$$

holds for all $x, y \in I$ and $t \in[0,1]$.

We will denote by $M P(I)$ the class of all multiplicatively $P$-functions on interval $I$. Clearly, $f: I \rightarrow[0, \infty)$ is multiplicatively $P$-function if and only if $\log f$ is $P$-function.

Remark. The range of the multiplicatively $P$-functions is greater than or equal to 1 .

Proof. Using the definition of the multiplicatively $P$-function, for $t=1$;

$$
f(x) \leq f(x) f(y) \Longrightarrow f(x)[1-f(y)] \leq 0 .
$$

Here, $f(x) \geq 0$, so we obtain $f(y) \geq 1$. Similarly, for $t=0$,

$$
f(y) \leq f(x) f(y) \Longrightarrow f(y)[1-f(x)] \leq 0 .
$$

Since $f(y) \geq 0$, we get $f(x) \geq 1$.

Example 1. The function $f:[0, \infty) \rightarrow[1, \infty), f(x)=x$ is a multiplicatively $P$-function. Really, for $x<y$, since $t x+(1-$ $t) y \leq y \leq x y$, we say that $f(x)=x$ is a multiplicatively $P$-function. 
Example 2. The function $f: \mathbb{R} \rightarrow[1, \infty), f(x)=|x|+1$ is a multiplicatively $P$-function.

$$
\begin{aligned}
f(t x+(1-t) y) & =|t x+(1-t) y|+1 \\
& \leq t|x|+(1-t)|y|+1 \\
& =t|x|+(1-t)|y|+t+1-t \\
& =t(1+|x|)+(1-t)(1+|y|) \\
& \leq t(1+|x|)(1+|y|)+(1-t)(1+|y|)(1+|x|) \\
& =(1+|x|)(1+|y|) \\
& =f(x) f(y)
\end{aligned}
$$

Example 3. The function $f:[0, \infty) \rightarrow[1, \infty), f(x)=e^{x}$ is a multiplicatively $P$-function. Really, for $\forall x, y \in[0, \infty)$ since $t x+(1-t) y \leq x+y$ we have

$$
f(t x+(1-t) y)=e^{t x+(1-t) y} \leq e^{x+y}=e^{x} e^{y}=f(x) f(y) .
$$

Remark. Let $f: I \rightarrow[1, \infty)$. Since $f(t x+(1-t) y) \leq t f(x)+(1-t) f(y) \leq f(x) f(y)$, every convex function whose range is $[1, \infty)$ is also multiplicatively $P$-function.

Theorem 2. Let $f, g: I \rightarrow[1, \infty)$. If $f$ and $g$ are multiplicatively $P$-function, then $f g$ are multiplicatively P-function.

Proof. For $x, y \in I$ and $t \in[0,1]$, we have

$$
\begin{aligned}
(f g)(t x+(1-t) y) & =f(t x+(1-t) y) g(t x+(1-t) y) \\
& \leq[f(x) f(y)][g(x) y(y)] \\
& =[f(x) g(x)][f(y) g(y)] \\
& =[(f g)(x)][(f g)(y)]
\end{aligned}
$$

This completes the proof of theorem.

Theorem 3. Let $f, g: I \rightarrow[1, \infty)$. If $f$ is multiplicatively $P$-function and decreasing and $g$ is convex function, then fog is multiplicatively P-function.

Proof. For $x, y \in[1, \infty)$ and $t \in[0,1]$, we obtain

$$
(f \circ g)(t x+(1-t) y)=f(g(t x+(1-t) y)) \leq f(\operatorname{tg}(x)+(1-t) g(y)) \leq f(g(x)) f(g(y))=(f \circ g)(x)(f \circ g)(y)
$$

This completes the proof of theorem.

\section{Hermite-Hadamard type inequalities for multiplicatively $P$-functions}

The goal of this paper is to develop concept of the multiplicatively $P$-functions and to establish some inequalities of Hermite-Hadamard type for these classes of functions.

Theorem 4. Let the function $f: I \rightarrow[1, \infty)$, be a multiplicatively P-function and $a, b \in I$ with $a<b$. If $f \in L[a, b]$, then the following inequalities hold:

(i) $f\left(\frac{a+b}{2}\right) \leq \frac{1}{b-a} \int_{a}^{b} f(x) f(a+b-x) d x \leq[f(a) f(b)]^{2}$.

(ii) $f\left(\frac{a+b}{2}\right) \leq f(a) f(b) \frac{1}{b-a} \int_{a}^{b} f(x) d x \leq[f(a) f(b)]^{2}$. 
Proof. (i) Since the function $f$ is a multiplicatively $P$-function, we write the following inequality:

$$
f\left(\frac{a+b}{2}\right)=f\left(\frac{[t a+(1-t) b]+[t b+(1-t) a]}{2}\right) \leq f(t a+(1-t) b) f(t b+(1-t) a)
$$

By integrating this inequality on $[0,1]$ and changing the variable as $x=t a+(1-t) b$, then

$$
f\left(\frac{a+b}{2}\right) \leq \frac{1}{b-a} \int_{a}^{b} f(x) f(a+b-x) d x
$$

Moreover, a simple calculation give us that

$$
\int_{0}^{1} f(t a+(1-t) b) f(t b+(1-t) a) d t \leq[f(a) f(b)]^{2} .
$$

So, we get

$$
f\left(\frac{a+b}{2}\right) \leq \frac{1}{b-a} \int_{a}^{b} f(x) f(a+b-x) d x \leq[f(a) f(b)]^{2} .
$$

(ii) Similarly, as $f$ is a multiplicatively $P$-function, we write the following:

$$
f\left(\frac{a+b}{2}\right) \leq f(t a+(1-t) b) f(t b+(1-t) a) \leq f(a) f(b) f(t b+(1-t) a)
$$

Here, by integrating this inequality on $[0,1]$ and changing the variable as $x=t b+(1-t) a$, then, we have

$$
f\left(\frac{a+b}{2}\right) \leq f(a) f(b) \frac{1}{b-a} \int_{a}^{b} f(x) d x
$$

Since,

$$
\frac{1}{b-a} \int_{a}^{b} f(x) d x \leq f(a) f(b)
$$

we obtain

$$
f\left(\frac{a+b}{2}\right) \leq f(a) f(b) \frac{1}{b-a} \int_{a}^{b} f(x) d x \leq[f(a) f(b)]^{2}
$$

This completes the proof of theorem.

Remark. Above Theorem (i) and (ii) can be written together as follows:

$$
f\left(\frac{a+b}{2}\right) \leq \frac{1}{b-a} \int_{a}^{b} f(x) f(a+b-x) d x \leq f(a) f(b) \frac{1}{b-a} \int_{a}^{b} f(x) d x \leq[f(a) f(b)]^{2} .
$$

Proof. By integrating the following inequality on $[0,1]$, the desired result can be obtained:

$$
f\left(\frac{a+b}{2}\right) \leq f\left(\frac{A_{t}+A_{1-t}}{2}\right) \leq f\left(A_{t}\right) f\left(A_{1-t}\right) \leq f(a) f(b) f\left(A_{t}\right),
$$

where $A_{t}=t a+(1-t) b$.

Theorem 5. Let $f: I \rightarrow \mathbb{R}$ be a differentiable function on $I^{\circ}$ such that the function $\left|f^{\prime}\right|$ is multiplicatively P-function. Suppose that $a, b \in I$ with $a<b$ and $f^{\prime} \in L[a, b]$. Then the following inequality holds:

$$
\left|\frac{f(a)+f(b)}{2}-\frac{1}{b-a} \int_{a}^{b} f(x) d x\right| \leq \frac{(b-a)\left|f^{\prime}(a)\right|\left|f^{\prime}(b)\right|}{4}
$$


Proof. Using Lemma 1, since $\left|f^{\prime}\right|$ is multiplicatively $P$-function, we obtain

$$
\begin{aligned}
\left|\frac{f(a)+f(b)}{2}-\frac{1}{b-a} \int_{a}^{b} f(x) d x\right| & =\left|\frac{b-a}{2} \int_{0}^{1}(1-2 t) f^{\prime}(t a+(1-t) b) d t\right| \\
& \leq \frac{b-a}{2} \int_{0}^{1}|1-2 t|\left|f^{\prime}(t a+(1-t) b)\right| d t \\
& \leq \frac{b-a}{2}\left|f^{\prime}(a)\right|\left|f^{\prime}(b)\right| \int_{0}^{1}|1-2 t| d t \\
& =\frac{(b-a)\left|f^{\prime}(a)\right|\left|f^{\prime}(b)\right|}{4}
\end{aligned}
$$

where $\int_{0}^{1}|1-2 t| d t=\frac{1}{2}$. This completes the proof of theorem.

The corresponding version for powers of the absolute value of the derivative is incorporated in the following result.

Theorem 6. Let $f: I \rightarrow \mathbb{R}$ be a differentiable function on $I^{\circ}$. Assume $q \in \mathbb{R}, q>1$, is such that the function $\left|f^{\prime}\right|^{q}$ is multiplicatively $P$-function. Suppose that $a, b \in I$ with $a<b$ and $f^{\prime} \in L[a, b]$. Then the following inequality holds:

$$
\left|\frac{f(a)+f(b)}{2}-\frac{1}{b-a} \int_{a}^{b} f(x) d x\right| \leq \frac{b-a}{2}\left(\frac{1}{p+1}\right)^{\frac{1}{p}}\left|f^{\prime}(a)\right|\left|f^{\prime}(b)\right|,
$$

where $\frac{1}{p}+\frac{1}{q}=1$.

Proof. Let $a, b \in I$. By assumption, Hölder's integral inequality, Lemma 1 and the inequality

$$
\left|f^{\prime}(t a+(1-t) b)\right|^{q} \leq\left|f^{\prime}(a)\right|^{q}\left|f^{\prime}(b)\right|^{q}
$$

we have

$$
\begin{aligned}
\left|\frac{f(a)+f(b)}{2}-\frac{1}{b-a} \int_{a}^{b} f(x) d x\right| & \leq \frac{b-a}{2} \int_{0}^{1}|1-2 t|\left|f^{\prime}(t a+(1-t) b)\right| d t \\
& \leq \frac{b-a}{2}\left(\int_{0}^{1}|1-2 t|^{p} d t\right)^{\frac{1}{p}}\left(\int_{0}^{1}\left|f^{\prime}(t a+(1-t) b)\right|^{q} d t\right)^{\frac{1}{q}} \\
& =\frac{b-a}{2}\left(\frac{1}{p+1}\right)^{\frac{1}{p}}\left(\int_{0}^{1}\left|f^{\prime}(t a+(1-t) b)\right|^{q} d t\right)^{\frac{1}{q}} \\
& \leq \frac{b-a}{2}\left(\frac{1}{p+1}\right)^{\frac{1}{p}}\left(\int_{0}^{1}\left|f^{\prime}(a)\right|^{q}\left|f^{\prime}(b)\right|^{q} d t\right)^{\frac{1}{q}} \\
& =\frac{b-a}{2}\left(\frac{1}{p+1}\right)^{\frac{1}{p}}\left|f^{\prime}(a)\right|\left|f^{\prime}(b)\right|
\end{aligned}
$$

where $\int_{0}^{1}|1-2 t|^{p} d t=\frac{1}{p+1}$.

A more general inequality using Lemma 1 is as follows.

Theorem 7. Let $f: I \rightarrow \mathbb{R}$ be a differentiable function on $I^{\circ}$. Assume $q \in \mathbb{R}, q \geq 1$, is such that the function $\left|f^{\prime}\right|^{q}$ is multiplicatively $P$-function. Suppose that $a, b \in I$ with $a<b$ and $f^{\prime} \in L[a, b]$. Then the following inequality holds:

$$
\left|\frac{f(a)+f(b)}{2}-\frac{1}{b-a} \int_{a}^{b} f(x) d x\right| \leq \frac{b-a}{4}\left|f^{\prime}(a)\right|\left|f^{\prime}(b)\right| .
$$


Proof. Let $a, b \in I^{\circ}$. Since the function $\left|f^{\prime}\right|^{q}$ is a multiplicatively $P$-function, from Lemma 1 and the power-mean integral inequality, we have

$$
\begin{aligned}
\left|\frac{f(a)+f(b)}{2}-\frac{1}{b-a} \int_{a}^{b} f(x) d x\right| & \leq \frac{b-a}{2} \int_{0}^{1}|1-2 t|\left|f^{\prime}(t a+(1-t) b)\right| d t \\
& \leq \frac{b-a}{2}\left(\int_{0}^{1}|1-2 t| d t\right)^{1-\frac{1}{q}}\left(\int_{0}^{1}|1-2 t|\left|f^{\prime}(t a+(1-t) b)\right|^{q} d t\right)^{\frac{1}{q}} \\
& \leq \frac{b-a}{2}\left(\int_{0}^{1}|1-2 t| d t\right)^{1-\frac{1}{q}}\left(\int_{0}^{1}|1-2 t|\left|f^{\prime}(a)\right|^{q}\left|f^{\prime}(b)\right|^{q} d t\right)^{\frac{1}{q}} \\
& =\frac{b-a}{2}\left(\int_{0}^{1}|1-2 t| d t\right)^{1-\frac{1}{q}}\left(\int_{0}^{1}|1-2 t| d t\right)^{\frac{1}{q}}\left|f^{\prime}(a)\right|\left|f^{\prime}(b)\right| \\
& =\frac{b-a}{4}\left|f^{\prime}(a)\right|\left|f^{\prime}(b)\right| .
\end{aligned}
$$

This completes the proof.

Theorem 8. Let $f: I \rightarrow \mathbb{R}$ be a differentiable function on $I^{\circ}$. Assume $q \in \mathbb{R}, q>1$, is such that the function $\left|f^{\prime}\right|^{q}$ is multiplicatively P-function. Suppose that $a, b \in I$ with $a<b$ and $f^{\prime} \in L[a, b]$. Then the following inequality holds:

$$
\left|\frac{1}{b-a} \int_{a}^{b} f(x) d x-f\left(\frac{a+b}{2}\right)\right| \leq \frac{(b-a)}{2}\left(\frac{1}{p+1}\right)^{\frac{1}{p}}\left|f^{\prime}(a)\right|\left|f^{\prime}(b)\right|,
$$

where $\frac{1}{p}+\frac{1}{q}=1$.

Proof. Since the function $\left|f^{\prime}\right|^{q}$ is a multiplicatively $P$-function, from Lemma 2 and the Hölder's integral inequality, we have

$$
\begin{aligned}
\left|\frac{1}{b-a} \int_{a}^{b} f(x) d x-f\left(\frac{a+b}{2}\right)\right| \leq & (b-a) \int_{0}^{\frac{1}{2}} t\left|f^{\prime}(t a+(1-t) b)\right| d t+\int_{\frac{1}{2}}^{1}|t-1|\left|f^{\prime}(t a+(1-t) b)\right| d t \\
\leq & (b-a)\left[\left(\int_{0}^{\frac{1}{2}} t^{p} d t\right)^{\frac{1}{p}}\left(\int_{0}^{\frac{1}{2}}\left|f^{\prime}(t a+(1-t) b)\right|^{q} d t\right)^{\frac{1}{q}}\right. \\
& \left.+\left(\int_{\frac{1}{2}}^{1}|t-1|^{p} d t\right)^{\frac{1}{p}}\left(\int_{\frac{1}{2}}^{1}\left|f^{\prime}(t a+(1-t) b)\right|^{q} d t\right)^{\frac{1}{q}}\right] \\
\leq & (b-a)\left[\left(\int_{0}^{\frac{1}{2}} t^{p} d t\right)^{\frac{1}{p}}\left(\int_{0}^{\frac{1}{2}}\left|f^{\prime}(a)\right|^{q}\left|f^{\prime}(b)\right|^{q} d t\right)^{\frac{1}{q}}\right. \\
& \left.+\left(\int_{\frac{1}{2}}^{1}|t-1|^{p} d t\right)^{\frac{1}{p}}\left(\int_{\frac{1}{2}}^{1}\left|f^{\prime}(a)\right|^{q}\left|f^{\prime}(b)\right|^{q} d t\right)^{\frac{1}{q}}\right] \\
= & (b-a)\left[\left(\frac{1}{(p+1) 2^{p+1}}\right)^{\frac{1}{p}} \frac{\left|f^{\prime}(a)\right|\left|f^{\prime}(b)\right|}{2^{1 / q}}+\left(\frac{1}{(p+1) 2^{p+1}}\right)^{\frac{1}{p}} \frac{\left|f^{\prime}(a)\right|\left|f^{\prime}(b)\right|}{2^{1 / q}}\right] \\
= & \frac{(b-a)}{2}\left(\frac{1}{p+1}\right)^{\frac{1}{p}}\left|f^{\prime}(a)\right|\left|f^{\prime}(b)\right|,
\end{aligned}
$$

where $\int_{0}^{\frac{1}{2}} t^{p} d t=\int_{\frac{1}{2}}^{1}|t-1|^{p} d t=\frac{1}{(p+1) 2^{p+1}}$ 
Theorem 9. Let $f: I \rightarrow \mathbb{R}$ be a differentiable function on $I^{\circ}$. Assume $q \in \mathbb{R}, q>1$, is such that the function $\left|f^{\prime}\right|^{q}$ is multiplicatively $P$-function. Suppose that $a, b \in I$ with $a<b$ and $f^{\prime} \in L[a, b]$. Then the following inequality holds:

$$
\left|\frac{1}{b-a} \int_{a}^{b} f(x) d x-f\left(\frac{a+b}{2}\right)\right| \leq \frac{b-a}{4}\left|f^{\prime}(a)\right|\left|f^{\prime}(b)\right|,
$$

where $\frac{1}{p}+\frac{1}{q}=1$.

Proof. Since the function $\left|f^{\prime}\right|^{q}$ is a multiplicatively $P$-function, from Lemma 2 and the power-mean integral inequality, we obtain

$$
\begin{aligned}
\left|\frac{1}{b-a} \int_{a}^{b} f(x) d x-f\left(\frac{a+b}{2}\right)\right| \leq & (b-a) \int_{0}^{\frac{1}{2}} t\left|f^{\prime}(t a+(1-t) b)\right| d t+\int_{\frac{1}{2}}^{1}|t-1|\left|f^{\prime}(t a+(1-t) b)\right| d t \\
\leq & (b-a)\left[\left(\int_{0}^{\frac{1}{2}} t d t\right)^{1-\frac{1}{q}}\left(\int_{0}^{\frac{1}{2}} t\left|f^{\prime}(t a+(1-t) b)\right|^{q} d t\right)^{\frac{1}{q}}\right. \\
& \left.+\left(\int_{\frac{1}{2}}^{1}|t-1| d t\right)^{1-\frac{1}{q}}\left(\int_{\frac{1}{2}}^{1}|1-t|\left|f^{\prime}(t a+(1-t) b)\right|^{q} d t\right)^{\frac{1}{q}}\right] \\
\leq & (b-a)\left[\left(\frac{1}{8}\right)^{1-\frac{1}{q}}\left(\frac{\left|f^{\prime}(a)\right|^{q}\left|f^{\prime}(b)\right|^{q}}{8}\right)^{\frac{1}{q}}+\left(\frac{1}{8}\right)^{1-\frac{1}{q}}\left(\frac{\left|f^{\prime}(a)\right|^{q}\left|f^{\prime}(b)\right|^{q}}{8}\right)^{\frac{1}{q}}\right] \\
= & \frac{b-a}{4}\left|f^{\prime}(a)\right|\left|f^{\prime}(b)\right| .
\end{aligned}
$$

\section{Applications to special means}

In this section, we shall consider the means for arbitrary real numbers $\alpha, \beta, \alpha \neq \beta$. For shortness, we will use the following means:

$A(\alpha, \beta)=\frac{\alpha+\beta}{2}, \quad \alpha, \beta \in \mathbb{R} \quad$ (arithmetic mean)

$\bar{L}(\alpha, \beta)=\frac{\beta-\alpha}{\ln |\beta|-\ln |\alpha|}, \alpha, \beta \in \mathbb{R} \backslash\{0\} \quad$ (logarithmic mean)

Now, using the results of Section 3 we will give some applications to special means of real numbers.

Proposition 1. Let $a, b \in \mathbb{R}, 0 \leq a<b$. Then we have

$$
\left|A\left(e^{a}, e^{b}\right)-\bar{L}\left(e^{a}, e^{b}\right)\right| \leq \frac{b-a}{2}\left(\frac{1}{p+1}\right)^{\frac{1}{p}} e^{a+b}
$$

Proof. The assertion follows from Theorem 6 applied for $f(x)=e^{x}, x \geq 0$.

Proposition 2. Let $a, b \in \mathbb{R}, 0 \leq a<b$. Then, we have

$$
\left|A\left(e^{a}, e^{b}\right)-\bar{L}\left(e^{a}, e^{b}\right)\right| \leq \frac{b-a}{4} e^{a+b} .
$$

Proof. The assertion follows from Theorem 7 applied for $f(x)=e^{x}, x \geq 0$.

Proposition 3. Let $a, b \in \mathbb{R}, 0 \leq a<b$. Then, we have, for all $q>1$

$$
\left|\bar{L}\left(e^{a}, e^{b}\right)-e^{A(a, b)}\right| \leq(b-a)\left(\frac{1}{(p+1) 2^{p+1}}\right)^{\frac{1}{p}} e^{a+b}
$$


Proof. The assertion follows from Theorem 8 applied for $f(x)=e^{x}, x \geq 0$.

Proposition 4. Let $a, b \in \mathbb{R}, 0 \leq a<b, 0 \notin[a, b]$. Then, we have

$$
\left|\bar{L}\left(e^{a}, e^{b}\right)-e^{A(a, b)}\right| \leq \frac{b-a}{4} e^{a+b} .
$$

Proof. The assertion follows from Theorem 9 applied for $f(x)=e^{x}, x \geq 0$.

\section{Competing interests}

The authors declare that they have no competing interests.

\section{Authors' contributions}

All authors have contributed to all parts of the article. All authors read and approved the final manuscript.

\section{References}

[1] Dragomir, S.S. Pečarić, J. and Persson, L.E. Some inequalities of Hadamard Type, Soochow Journal of Mathematics, Vol.21, No:3, pp. 335-341, July 1995.

[2] Dragomir, S. S. and Agarwal, R. P. Two inequalities for di erentiable mappings and applications to special means of real numbers and to trapezoidal formula, Appl. Math. Lett. 11 (1998), 91-95.

[3] Dragomir, S.S. and Pearce, C.E.M. Selected Topics on Hermite-Hadamard Inequalities and Its Applications, RGMIA Monograph (2002).

[4] Hadamard, J. Etude sur les proprietes des fonctions entieres en particulier d'une fonction consideree par Riemann, J. Math. Pures Appl. 58, 171-215 (1893).

[5] Kirmaci U. S. Inequalities for differentiable mappings and applications to special means of real numbers and to midpoint formula, Applied Mathematics and Computation 147 (2004) 137-146

[6] Pečarić, J.E. Proschan, F. and Tong, Y.L. Convex Functions, Partial Orderings, and Statistical Applications, Academic Press Inc., 1992, p. 137.

[7] Zabandan, G. A new refinement of the Hermite-Hadamard inequality for convex functions, J. Inequal. Pure Appl. Math. 10(2), Article ID 45 (2009). 